\title{
An Object-Oriented Framework For Valuing Shout Options On High-Performance Computer Architectures
}

\author{
H. Windcliff*, K.R. Vetzal ${ }^{\dagger}$, P.A. Forsyth ${ }^{\ddagger}$, A. Verma ${ }^{\S}$, and T.F. Coleman $₫$
}

November 28, 1999

\begin{abstract}
A shout option is a financial contract which allows the holder to change the payoff during the lifetime of the contract. For example, the holder could have the right to set the strike price to the current value of the underlying asset. Complex versions of these options are embedded in financial products which offer various types of maturity guarantees such as segregated funds marketed by Canadian insurance companies. The value of these options can be determined by solving a collection of coupled partial differential equations (PDEs). In this work we develop an extensible, object-oriented framework for valuing these contracts which is capable of exploiting modern, high-performance supercomputing architectures. We use this framework to study and illustrate practical aspects of valuing and hedging these contracts.
\end{abstract}

Keywords: PDE option pricing, shout options, object-oriented, high-performance architecture

Running Title: Shout Options

Acknowledgement: This work was supported by the Natural Sciences and Engineering Research Council of Canada, the Social Sciences and Humanities Research Council of Canada, the Communications and Information Technology Office of Ontario, and the Financial Industry Solutions Center (FISC), an SGI/Cornell University Joint Venture. The authors would like to thank Josh Blumert, SGI Systems Engineer, for his friendly help and advice.

${ }^{*}$ Department of Applied Mathematics, University of Waterloo, Waterloo, Ontario, Canada, N2L 3G1, hawindcliff@elora.math. uwaterloo.ca

†Centre for Advanced Studies in Finance, University of Waterloo, kvetzal@watarts .uwaterloo.ca

- Department of Computer Science, University of Waterloo, paf orsyt@elora.math.uwaterloo.ca

$\S$ Financial Industry Solutions Center, New York, and Cornell Theory Center, Cornell University, Ithaca NY, U.S.A. 14853 , verma@cs . cornell .edu

IFinancial Industry Solutions Center, New York, and Computer Science Department, Cornell University, coleman@cs.cornell .edu 


\section{Introduction}

Many financial products marketed to investors contain embedded options. Familiar examples include callable bonds, convertible bonds, and savings bonds. Currently perhaps the most prominent example in Canada are investment funds which provide maturity guarantees. Frequently sold by life insurance companies, such products are known as "segregated funds". Contracts of this form give the investor the benefits of the higher returns common in the equity market while providing downside protection should the market fall. Although various types of these contracts have been around at least since the early 1960s, they have recently become very popular. ${ }^{1}$

The options which are contained in these types of contracts range from quite simple to extremely complex. For example, a straightforward set guarantee effectively provides the investor with a European put option. A more complicated case is a protective floor index, which allows the holder to set a minimum amount to be received at the maturity of the contract. A protective floor index effectively consists of the purchase of the stock index along with a shout put option. A shout option is an option which allows the holder to reset the strike price during the life of the contract. At maturity the value of the stock index together with the payment provided by the shout put option is always worth at least the protective floor level.

Some of the most complicated options are embedded in segregated funds. Such contracts provide death benefits if the investor dies before the maturity date in addition to complex maturity guarantees. Investors are often permitted to reset the level of the guarantee (i.e. "shout") multiple times, up to some limit within a time period (e.g. four times per year). When an investor shouts, the maturity date of the guarantee may (or may not) be extended. Shouting more than once may involve a reduced percentage guarantee. For example, the first shout may set the guarantee at the current index level, but shouting a second time may only provide something like $98 \%$ of the prevailing index level, while a third shout might provide a protection level of $96 \%$.

Accurate models for the valuation and hedging of these types of options are required for at least two reasons. First, it is important for both individual investors and the firms selling these complex contracts to have good estimates of the value of the included option features. Second, it is critical for the institutions offering such products to be able to hedge the risk exposures involved. ${ }^{2}$

There has not been much academic research in this general area. Simple maturity guarantees have been explored in [4]. Various (but relatively uncomplicated) kinds of shout options have been described in [12] and [6]. Both of these papers describe the valuation using a binomial or trinomial lattice method, and describe similarities between these type of options and various other exotic options. Perhaps the most obvious point of comparison is to a lookback option. If the holder happens to shout when the underlying asset price is at

\footnotetext{
${ }^{1}$ Net new sales of segregated funds in Canada grew from $\$ 701$ million in 1996 to $\$ 5.9$ billion in 1998 , while the number of segregated funds being offered increased from 246 to more than 800 (The Globe and Mail, October 23, 1999).

${ }^{2}$ For a brief description of some concerns which have been raised regarding the pricing and hedging of these contracts, see W. Falloon, "Canada's option nightmare", RISK, August 1999.
} 
its minimum (for a call) or maximum (for a put) value during the life of the contract, then the option's payoff will be that of a lookback. As noted in [12], this implies that the value of a lookback is an upper bound for the value of a shout option. Alternatively, as pointed out in [6], shout options can be viewed as a restricted version of lookback options: if the holder of a shout option has an infinite number of shout opportunities, he will shout whenever the underlying asset reaches a new maximum (in the case of a put) or minimum (for a call), thereby effectively creating a lookback. More interestingly, if there is no initial floor level and only a single shout opportunity, it is shown in [6] that the optimal shout policy for the holder is deterministic, depending only on time (and not on the future level of the underlying asset).

With regard to valuation techniques, note that in [6] the authors work under the standard Black-Scholes assumptions and deal exclusively with shout options which reset the strike price to the current value of the underlying asset. These simplifications reduce the dimensionality of the numerical problem. In this paper we consider more complex types of shout options, while making minimal assumptions about the behaviour of the underlying asset. To achieve this, we employ a numerical PDE approach. This offers several potential benefits:

- Using a fully numerical approach allows for more general specifications of volatility than the basic geometric Brownian motion assumption of Black-Scholes. Examples include CEV models [8] and implied volatility surfaces [1, 7].

- Best/worst case uncertain parameter (e.g. volatility, interest rate, dividend yield) models $[2,15]$ can be used. Such models may be particularly suited to this type of application, because the contracts are typically quite long term and have complicated provisions. Uncertain parameter models can provide a reasonable compromise between the added realism of additional stochastic factor(s) and the tractability of a single stochastic factor framework.

- If desired, extensions to features such as discrete dollar dividends or barrier-type provisions can be easily accommodated. Discrete dollar dividends are of interest because the payment of the option is often amortized discretely over the life of the contract. Barrier features are one possible means of incorporating default risk into the valuation of these contracts.

- Since these contracts are typically long term the second order rate of convergence of PDE methods is of practical interest when compared with the first order convergence of lattice techniques.

It is necessary to develop robust, extensible algorithms for valuing these contracts. It will be seen that the value of a shout option can be determined by valuing a collection of simpler contracts. Object oriented computer languages allow us to create a class library which will permit maintainability through code reuse. Further, by careful design and encapsulation of data, it is easy to modify the program to take advantage of modern multiprocessor computer architectures. 


\section{Some Background Details}

For clarity, we follow [16] and provide this definition of a shout option:

Definition: A shout option is a contract defined by the following objects:

- An underlying asset price process $S$ upon which the derivative security is written.

- A maturity time $T$ for the contract.

- A payoff function $g(S, K, U)$ which determines the payment made to the holder of the security at maturity which is a function of the asset level $S$ and a parameter called the strike, $K$, which can be changed (at the discretion of the holder) during the life of the contract.

- A maximum number of times $U_{\max }$ which the holder of the security can shout during a given time period, thereby resetting the strike $K$. We will use the discrete variable $U=0, \ldots, U_{\max }$ to count the number of shouts used at any point in time.

- A function $\mathcal{F}(S, K, U, t)$ which determines how the strike $K$ is set upon shouting. In the case of a simple shout option where the strike is reset to the current asset level, this function would be defined as $K^{*}=S$.

- A shout dividend function $\mathcal{D}(S, K, U, t)$ which represents payments generated by the option upon shouting. Cases where $\mathcal{D}(S, K, U, t)<0$ can be thought of as a fee charged for shouting.

From this it can be seen that the holder of a shout option has the ability to improve on the contract presently held by choosing to reset the strike $K$ if it is beneficial, in exchange for reduced future flexibility. This is a type of American option, where the payoff upon exercise is specified by the function $\mathcal{D}(S, K, U, t)$, along with the the value of the contract received. It is also worth noting that the function $\mathcal{F}(S, K, U, t)$ could be multidimensional in some cases, such as contracts where shouting sets both a new floor level and a new expiry time.

We assume that there is a risk free money market account and that the price of the underlying asset $S$ satisfies the stochastic differential equation

$$
d S=\mu(S, t) S d t+\sigma(S, t) S d z
$$

where $d z$ is the increment of a Wiener process and $\mu(S, t)$ and $\sigma(S, t)$ are the drift rate and volatility respectively. Standard no-arbitrage arguments then allow us to model the value of the shout option as

$$
\begin{aligned}
\frac{\partial V}{\partial t}+(r(t)-q(t)) S \frac{\partial V}{\partial S}+\frac{1}{2} \sigma(S, t)^{2} S^{2} \frac{\partial^{2} V}{\partial S^{2}}-r(t) V & \leq 0 \\
V^{*} & \leq V
\end{aligned}
$$

where at least one of (1-2) holds with equality throughout the domain. Here $r(t)$ is the risk free rate of return, $q(t)$ is the dividend yield on the underlying asset, $V=V(S, K, U, t)$, and 
we have defined $V^{*}$ (the value of the contract the holder receives upon shouting) as

$$
V^{*}(S, K, U, t)= \begin{cases}V(S, \mathcal{F}(S, K, U, t), U+1, t)+\mathcal{D}(S, K, U, t) & \text { if } U+1 \leq U_{\max } \\ -\infty & \text { otherwise }\end{cases}
$$

The terminal condition

$$
V(S, K, U, T)=g(S, K, U)
$$

is imposed at contract expiry $t=T$.

Following [16], we can express (1-2) in terms of an equivalent penalty method [17]:

$$
\frac{\partial V}{\partial t}+(r(t)-q(t)) S \frac{\partial V}{\partial S}+\frac{1}{2} \sigma(S, t)^{2} S^{2} \frac{\partial^{2} V}{\partial S^{2}}-r(t) V=-Q\left(V, V^{*}\right)
$$

where the penalty term $Q$ serves to enforce the relevant constraints. This formulation is used in our actual computations. Some other aspects of these valuation problems worth noting are:

- In many cases, the holder is permitted to shout up to a maximum of $U_{\max }$ times during a given time period (usually one year). At the end of this period, the discrete variable $U$ which tracks the number of shout opportunities used is reset to zero. If $t_{i}$ is a shout counter reset time, then absence of arbitrage requires that the jump condition

$$
V\left(S, K, U, t_{i}^{-}\right)=V\left(S, K, 0, t_{i}^{+}\right)
$$

must hold for all $U$ where $t_{i}^{-}$and $t_{i}^{+}$are the times immediately before and after $t_{i}$ respectively.

- Under Black-Scholes modelling assumptions (i.e. $\sigma(S, t)=\sigma_{B S}$, a constant), the dimensionality of the problem can be reduced for some contract specifications by working with a new variable $\zeta=S / K$. See [16] for further details about this similarity solution.

- Situations where $\mathcal{F}(S, K, U, t)$ is multidimensional add complexity to the problem. For example, a common feature with segregated funds is that shouting involves resetting not only the strike but also the time to expiry. In this case, an extra dimension is required for a variable to track the expiration time of the contract.

In general (ignoring the case where $\mathcal{F}(S, K, U, t)$ is multidimensional to simplify the discussion), we are faced with a three dimensional problem. The three dimensional system of PDEs is first discretized in time, using a Crank-Nicolson method. Recall that the variable $U$ represents the number of shouts used by the holder and so it can only take on discrete integer values $U=0,1, \ldots, U_{\max }$. Within each plane $U=$ const., the variable $K$ is discretized $K=K_{0}, \ldots, K_{\max }$. Finally, for each value of $U=$ const., $K=$ const., the one dimensional PDE equation (5) is discretized using a finite volume method [16]. Note that typically we have to interpolate the discrete solution in order to determine the value of the option received upon shouting, as specified in equation (3).

There are a number of significant numerical issues involved in valuing these contracts. These include constructing an efficient grid, the treatment of boundary conditions, choice of interpolation method, and method of enforcing the constraint $V^{*}$ (equation (3)). We will not discuss these matters here, referring interested readers to a companion paper [16] which provides a thorough treatment and a detailed validation of the algorithms. 


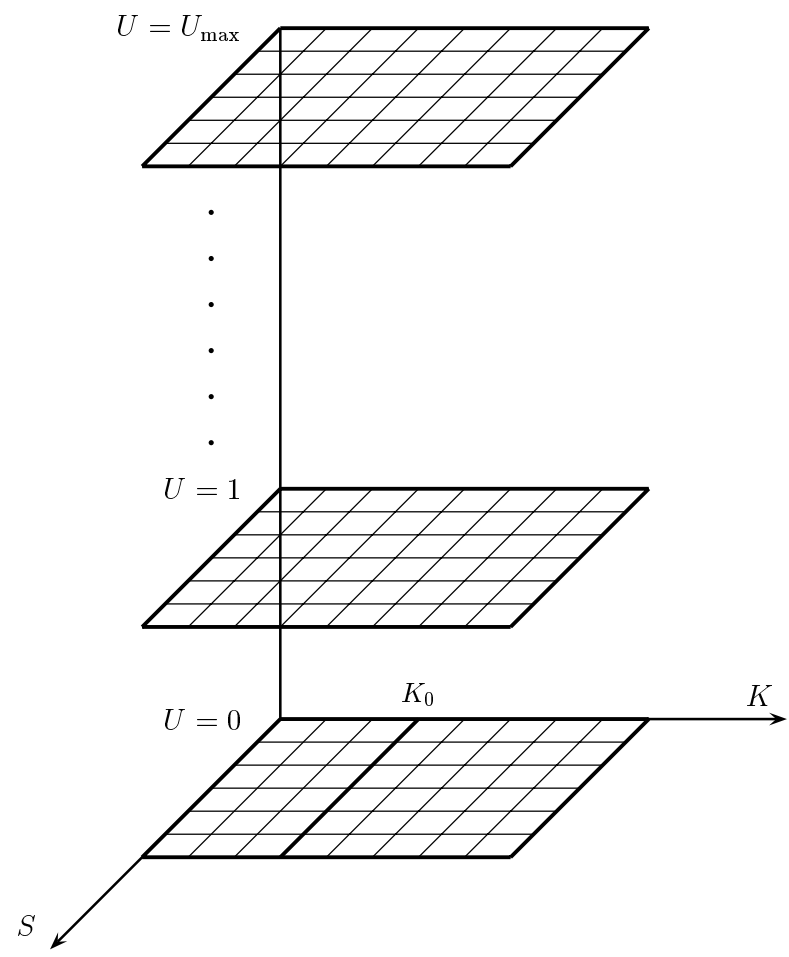

FiguRE 1: An object-oriented interpretation of a shout option. The line $K=K_{0}$ represents the contract that was initially sold to the investor. We define the object type ShoutPlane to represent planes with $U=$ const. Each ShoutPlane will contain a number of OneD objects which represent a line of $K=$ const. within a plane of $U=$ const.

\section{An Object-Oriented Implementation}

Recall that the value of the contract depends on the four independent variables $S, K, U$, and $t$. We are ultimately interested in the value of a shout option for a given level of the underlying asset $S_{0}$ with a specific initial strike $K_{0}$ and with no shouts used $\left(U_{0}=0\right)$ at the time of sale of the security. This problem is a three dimensional, time-dependent non-linear differential equation (5).

However, this PDE can be simplified by exploiting the structure of the dependence on the variables $U$ and $K$. For example, note that equation (5) depends on $(K, U)$ only through the penalty term $Q\left(V, V^{*}\right)$. If we imagine solving the PDE (5) by stepping through time, we can see that once the solution has been obtained for a given value of $U$, then this determines the value of $V^{*}$ for all discrete values of $K$ for the plane $U-1$. Consequently, at each discrete timestep, we solve for each plane of $U=$ const. in the order $U_{\max }, U_{\max }-1, . ., 0$. Within each plane $U=$ const., the one dimensional PDEs for different discrete values of $K$ are completely independent, since $V^{*}$ for this plane is known from the solution in plane $U+1$.

Recall that the discrete variable $U$ represents the number of shouts currently expended. Therefore, planes of $U=$ const. represent contracts which have the same number of shouts used (see Figure 1). It is convenient to define an object type ShoutPlane which encapsulates planes of $U=$ const. We reiterate here that, within each timestep, we compute solutions 
in the order $U_{\max }, U_{\max }-1, \ldots, 0$. As a result, within the plane $U=$ const., we have a set of independent one dimensional PDEs. This framework of objects which encapsulate planes of $U=$ const. allows us to internally schedule many aspects of the solution process such as the timestepping algorithm. This will be helpful in a later section when we discuss the parallelization of the algorithm. This also gives us the ability to define generic operations such as interpolation and data manipulation easily and safely. For example, often our algorithm will require information not contained exactly within our discrete mesh. Defining these objects in an abstract manner allows us to define operations such as interpolation,

double ShoutPlane: interpolate( double S, double K );

giving us the ability to externally query the object in a manner naturally defined by the contract. The user of these functions need not be concerned with any of the details of the $S$ and $K$ discretizations. In fact, there may not even be a discretization for one of the state variables; for example, in the case of the similarity reduction mentioned above. When using a similarity reduction, the ShoutPlane maintains only a single line of $K=$ const. and the interpolation member function is defined appropriately. This improves the maintainability of the software by allowing the same interface to be used for the ShoutPlane regardless of the implementation details chosen.

Within each ShoutPlane, the line $K=$ const. represents a particular setting of the strike price. The solution along each of these lines will be encapsulated in a 0neD object. As a result, each of the 0neD objects contains both the $S$ discretization and the solution for a particular strike setting. These 0 neD objects are then given methods to set minimum constraints, interpolate, and solve the partial differential equation (5) using techniques described in [16]. In fact, it can be seen that these objects are merely solving equations modelling a standard American option with a time varying constraint. It should be noted that many exotic options can be formulated in a similar fashion, i.e. in terms of a collection of these building block contracts. Some examples are provided in $[18,14]$. Therefore, a robust implementation of the OneD object can allow algorithms for these complex options to be developed efficiently through code reuse.

So far we have discussed a structure for maintaining the data which must be available to determine the value of a shout option. We must also be able to control and schedule the communication of this data. For this we define a scheduler/controller object which is responsible for scheduling the solution of the building block objects and determining the minimum value constraint $V^{*}$. Each of the planes of constant $U$ must be solved for all strike prices $K$ and asset prices $S$ on the grid and advanced (backwards) through time using a timestepping algorithm. Should the holder of the security shout, they receive a security defined on the next higher plane $U^{*}=U+1$ with strike $K^{*}=\mathcal{F}(S, K, U, t)$. Consequently, the controller class schedules the ShoutPlane objects in the order $U=U_{\max }, \ldots, 0$.

Observe that upon shouting information is collected from the plane one level above the current position according to the "look up function" $\mathcal{F}$. The value is only reset to $V^{*}$ in the case that $V^{*} \geq V$. This effectively sets an American-type floor to the value of the contract which is imposed continuously through time. The scheduler/controller can be implemented in the following manner: 
For each $U=0, \ldots, U_{\max } / /$ initialization

ShoutPlane $[U]=$ new ShoutPlane

For each discrete $K$

ShoutPlane [U].0neD [K] = new OneD

ShoutPlane $[\mathrm{U}] .0 \mathrm{neD}[\mathrm{K}] \cdot \mathrm{value}=\operatorname{payof} f(\mathrm{~K}, \mathrm{U})$

End for $K$

End for $U$

$t:=T / /$ timestep loop

While $t>0$ do

$t=\max (t-\Delta t, 0)$

For $U=U_{\max }, \ldots, 0$

For each discrete $K$

ShoutPlane $[\mathrm{U}]$. OneD $[\mathrm{K}]$. constraint $=$ get_constraint $(\mathrm{K}, \mathrm{U}, \mathrm{t})$

ShoutPlane [U] .0neD [K] . advance_solution()

End for $K$

End for $U$

End while

To illustrate further, consider the case of a standard shout put option where the strike level is reset to the current asset price upon shouting. In this case for any $S$ the controller determines the constraint by looking at the value of the contract on the diagonal $K^{*}=S$ on the next higher plane $U+1$ (see Figure 2).

\section{A Parallel Version for Multiprocessor Architectures}

If we are interested in utilizing a more complicated specification than the standard BlackScholes setting with constant volatility, we must solve the full three dimensional problem. Other cases where we cannot use the similarity solution include some types of contract specifications and uncertain parameter models. In these cases, the complexity of the numerical solution may become so large that we cannot solve these problems quickly enough on a uniprocessor machine.

Using our object-oriented construction described above, we note that the valuation of the option for fixed $U$ and $K$ is independent of other values of $U$ and $K$ except through the application of the constraint $V^{*}(S, K, U, t)$ defined in (3). As a result, each of the OneD objects in a given plane of $U=$ const. can be solved independently once the minimum value constraint $V^{*}$ has been determined by the controller class.

In this work, we use the OpenMP library. This library is widely available on many multiprocessor machines, and allows for development of portable software. The OpenMP library consists of a set of preprocessor compiler directives (pragmas) which are ignored on single processor architectures. We emphasize here that no changes were made to the basic algorithms or software for these tests. We simply added the compiler directives in a small number of locations. 


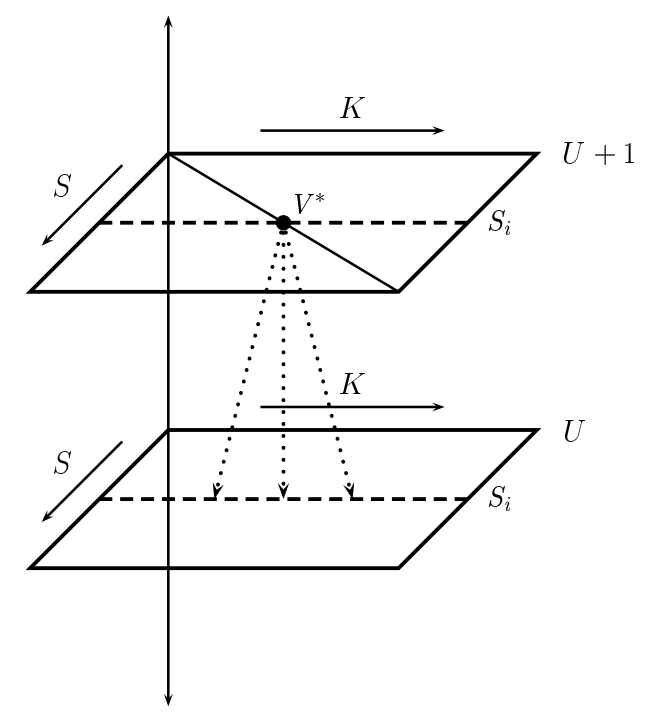

FiguRE 2: The flow of information in a standard shout option with $\mathcal{F}(S, K, U, t)=S$. The controller class determines the minimum value constraint by interpolating the solution on the next higher ShoutPlane. Notice that all the values along the line $S=S_{i}$ are compared with the same constraint value $V^{*}=V\left(S_{i}, S_{i}, U+1, t\right)$ to determine whether or not shouting is optimal.

Since all of the data has been safely encapsulated within the OneD objects, we can easily parallelize the code without worrying about potential data sharing problems. The OpenMP library allows us to allocate multiple threads for parallel regions by simply specifying appropriate pragma calls. Since the one dimensional PDE problems all require approximately the same number of floating point operations, we find that static scheduling affords the best performance. Further, we allocate chunksizes of 8 iterations through the loop since this improves the performance of the caching system. The following is a code fragment which implements the parallelization of the advance_solution section of the pseudo code (7) given above.

void ShoutPlane: :advance_solution()

\{

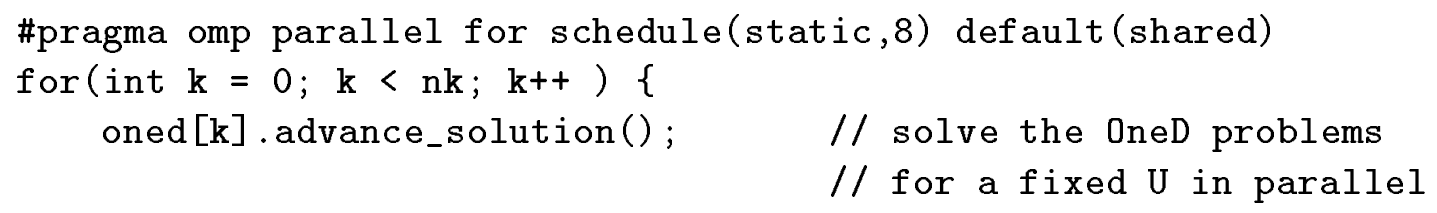

\}// end ShoutPlane: :advance_solution() 


\begin{tabular}{|l|cccc|}
\cline { 2 - 5 } \multicolumn{1}{c|}{} & \multicolumn{4}{c|}{ Number of Processors } \\
\cline { 2 - 5 } \multicolumn{1}{c|}{} & 2 & 4 & 8 & 16 \\
\hline$K$ discretization & \multicolumn{4}{c|}{ speed up } \\
\hline \hline 128 nodes & 1.94 & 3.69 & 7.07 & 12.08 \\
\hline 256 nodes & 1.95 & 3.68 & 6.96 & 12.65 \\
\hline Theoretical & 1.96 & 3.77 & 7.02 & 12.31 \\
\hline
\end{tabular}

TABLE 1: Timing results for parallel version of algorithm on SGI Origin 2000 cc-NUMA multi-processor server; speed up $=\frac{\text { time serial }}{\text { time parallel }}$. The number of nodes in the $K$ discretization gives the number of independent problems on each plane of $U=$ const. The last row gives the theoretical speed up using (9) assuming $98 \%$ parallelization. The shout option has four exercise opportunities per year and a five year maturity. Black-Scholes modelling assumptions with $\sigma_{B S}=.2, r=.1, K_{0}=\$ 100$. These results are for a three dimensional case (although a similarity solution exists for this particular problem, we did not use it in these computations).

Code fragment (8) indicates that the addition of multithreading directives to the software is not an onerous task.

If we study the serial version of implementation of the pseudo code, we find that approximately $81 \%$ of the processor time is spent advancing the solution and $17 \%$ of the time is spent interpolating the numerical solution to determine the constraint $V^{*} \cdot{ }^{3}$ Both of these sections of the software can be parallelized using a few compiler directives similar to fragment (8). We did not attempt to expose any other parallelism in this algorithm, being satisfied with $98 \%$. Since both the time advance and interpolation sections can be parallelized, we can estimate the maximum theoretical speed up using the formula:

$$
\text { speed up }=\frac{100}{\frac{98}{N}+2}
$$

where $N$ denotes the number of processors used. Table 1 shows typical results for a full three dimensional shout option valuation. As can be seen from the results, the observed increase in speed is very close to the theoretical limit in equation (9).

\section{$5 \quad$ Illustrative Examples}

\subsection{General Features}

We begin by providing some results for the Black-Scholes model case where the holder has five opportunities during the life of the contract to reset the strike of a put option to the prevailing value of the underlying asset. These examples also assume that there is an initial strike $K_{0}$ set at $\$ 100$. Table 2 presents results for contract lengths of five, ten, and twenty years. Although these contracts are quite valuable, they are fairly insensitive to the time to expiry. The at the money contract with $S=\$ 100$ increases from $\$ 24.37$ when $T=5$ years

\footnotetext{
${ }^{3}$ The interpolation is expensive since we solve an inverse problem to determine mesh points to use in the diagonal interpolation described in [16] which dramatically improves convergence.
} 


\begin{tabular}{|l|r|r|r|}
\cline { 2 - 4 } \multicolumn{1}{c|}{} & \multicolumn{3}{|c|}{ Shout Option Values } \\
\cline { 2 - 4 } \multicolumn{1}{c|}{} & $T=5$ years & $T=10$ years & $T=20$ years \\
\hline$S=\$ 80$ & 23.587 & 24.492 & 23.307 \\
\hline$S=\$ 100$ & 24.374 & 27.280 & 27.466 \\
\hline$S=\$ 120$ & 27.659 & 31.175 & 31.975 \\
\hline
\end{tabular}

TABLE 2: Similarity solution for the value of a shout put option with five exercise opportunities. Black-Scholes modelling assumptions with $\sigma_{B S}=.25, r=.06$, and $K_{0}=\$ 100$. Values were obtained using a grid with 800 nodes. More detailed results illustrating execution timing and convergence for this case are provided in [16].

to $\$ 27.28$ when $T=10$ years, but only marginally further to $\$ 27.47$ when $T=20$ years. The contract values exhibit similar behaviour for the $S=\$ 120$ case. However, when $S=\$ 80$, the 20 year contract is less valuable than the 10 year contract. One advantage of our PDE approach is that we use an automatic timestep size selector, similar to that described in [10]. Consequently, the longer term options are not much more expensive computationally than the shorter term ones (uniprocessor CPU times are 259, 404, and 529 seconds for the five, ten, and twenty year options respectively ${ }^{4}$ ). This is because the solution becomes smoother as time evolves, and accordingly the automatic timestep selector takes bigger timesteps. This type of efficiency gain for long term options is not possible with lattice type methods.

Consider a standard shout put option where the holder of the security has the ability to reset the strike price to the current asset level. Since the holder can always choose not to reset the strike from its initial setting, these contracts must always be worth at least as much as a European put option with the same initial strike as shown in Figure 3(a). As the asset price $S \rightarrow \infty$ it becomes profitable for the holder of the shout put option to reset the strike to the higher asset level since the current strike setting is unlikely to be in-the-money at expiry. In Figure 3(b) we can see that as $S \rightarrow \infty$ the shout put options with various initial strike settings all tend asymptotically to the same value. However, under the market conditions in this example, there is no optimal exercise boundary at five years to maturity. ${ }^{5}$ Therefore, the values for the different strikes do not become identical no matter how large the value of $S$ since in all cases it is optimal to hold on to the opportunity to shout. The small differences remaining for large $S$ are simply differences in the values of extremely deep out of the money options.

Figure 4 represents a case where there is an optimal exercise boundary. The figure illustrates how the algebraic constraints interact with the solution of the equation. The solid line represents the value of a shout put option with a single exercise opportunity which has a current strike set at $K_{0}=\$ 100$. The dotted lines represent the values of standard European put options with various strike settings; these are the solutions for particular $K=$ const. on the $U+1$ plane. Notice how the value of the shout put option is required to lie above the at the money value of each of the European options since the owner of the shout can

\footnotetext{
${ }^{4}$ This is for a fine grid with 800 nodes. As shown in [16], reasonably accurate results can be obtained using much coarser grids. For example, using a grid with 100 nodes results in pricing errors of about 5 cents. This requires around 5 seconds of CPU time for the 20 year case.

${ }^{5}$ For a more detailed discussion regarding the optimal exercise boundary, refer to [16].
} 


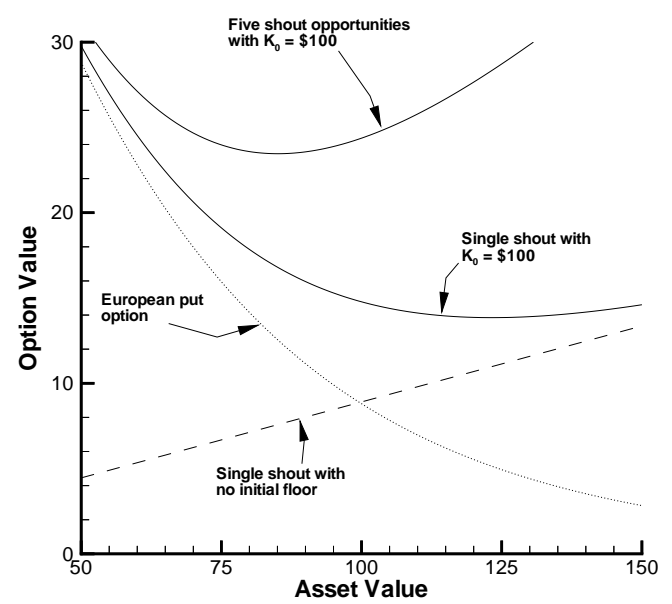

(a) Shout put options with one and five shout opportunities and initial floor set at $K_{0}=\$ 100$. Also shown are a European put option with $K=\$ 100$ and a shout put option with no initial floor setting.

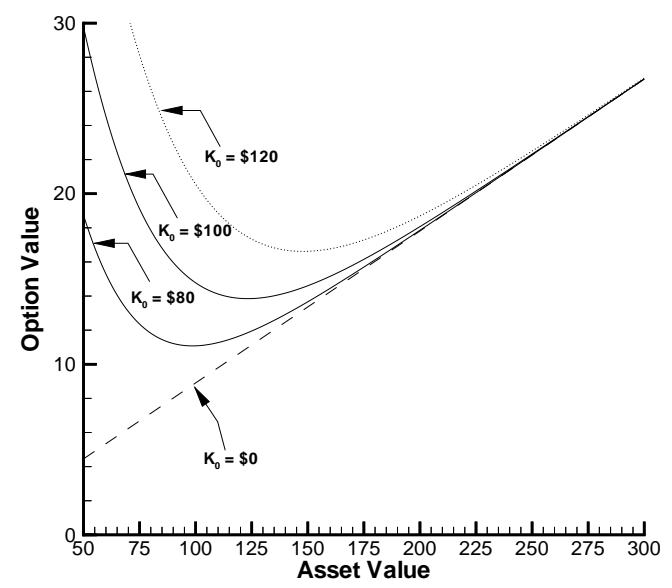

(b) Shout put options with various initial strike settings. Each option has a single exercise opportunity.

FiguRE 3: Comparison of various shout options. Black-Scholes modelling assumptions with $\sigma_{B S}=.25, r=.06$, and $T=5$ years.

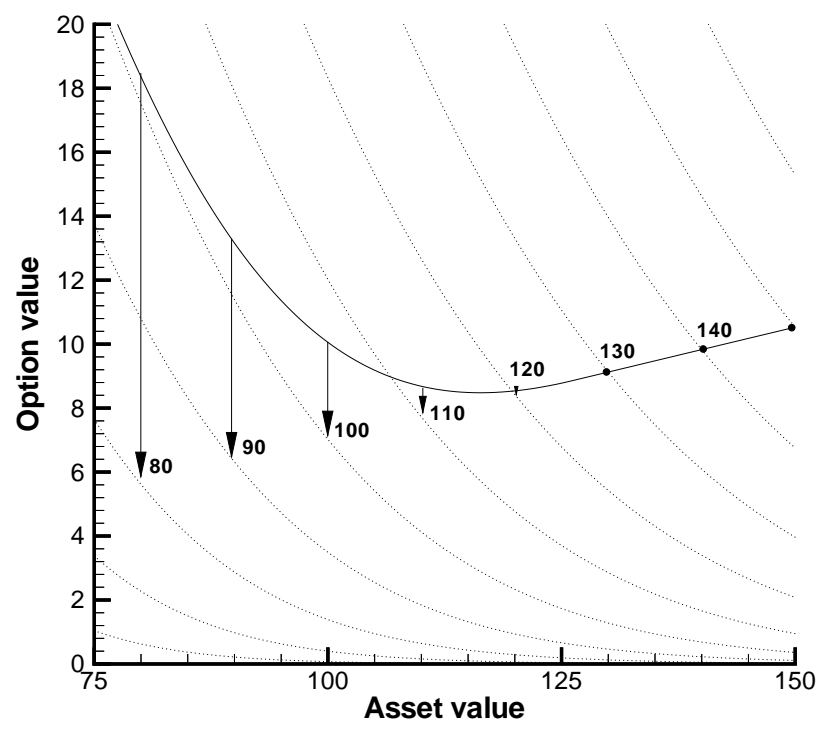

FIGURE 4: Value of a shout put option with a single exercise opportunity and initial strike $K_{0}=\$ 100$ (solid), compared with the value of at the money European put options (dotted). The arrows denote the change in value of the shout option upon shouting. Black-Scholes modelling assumptions with $\sigma_{B S}=.25, r=.06$, and $T=1$ year. 


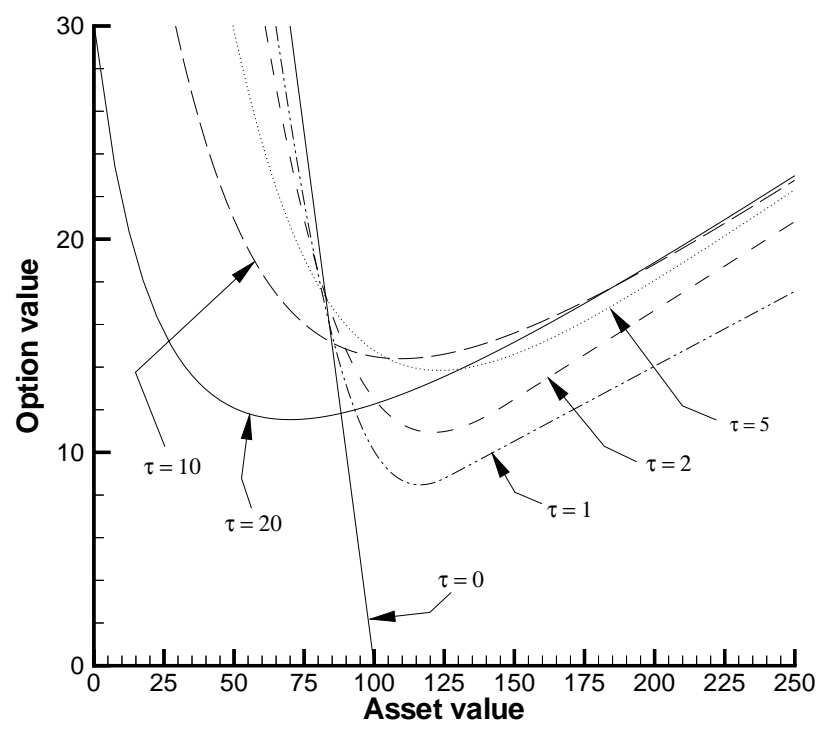

FIgURE 5: Value of a shout put option with a single shout opportunity and an initial strike $K_{0}=\$ 100$ for various times $\tau$ remaining until maturity. Black-Scholes modelling assumptions with $\sigma_{B S}=.25$ and $r=.06$.

receive these contracts by shouting. For asset levels above $S \approx \$ 130$ the holder receives a security which is worth the same amount as the shout security. This is the exercise region, where it is optimal for the holder to shout. For lower asset values, the holder would receive a security which is worth less than the shout security which he currently owns. Therefore in this region it is optimal for the holder not to shout.

Figure 5 plots the value of a shout option for various times remaining until maturity. With a long time left, the option value profile is roughly U-shaped. As the time until maturity gets relatively short, the profile gradually becomes more V-shaped, though of course it collapses to a standard European put payoff at expiry. One implication of this behaviour is that it may become relatively more difficult to hedge these options as they approach maturity.

As noted above, many options embedded in products sold to Canadian investors also feature a maturity extension upon shouting. In other words, the investor is permitted to reset the guarantee level, but when this is done the time for which the guarantee applies is extended. Figure 6 plots the option value for a typical case where the investor originally has a ten year (European) put option with a strike price of $\$ 100$. Upon shouting, the investor would effectively receive an at the money put option which expires ten years from that time (not from when the contract was originally bought). There is a maximum possible maturity of 30 years from the original purchase date (i.e. shouting is only allowed for a period of 20 years from the initial sale date). In this case, we are solving a three dimensional problem (it would be four dimensional if not for the similarity reduction). The option value profile is broadly similar to that of a standard shout where only the strike is reset. Comparing Figures 6 and 7, it can be seen that the value of a contract permitting two shouts per year 


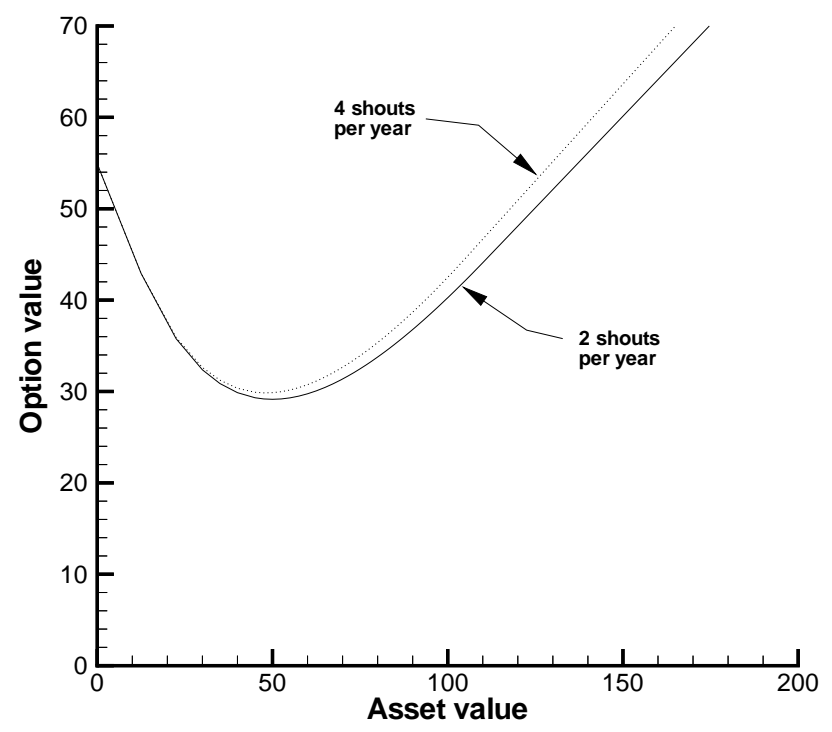

FIGURE 6: Value of shout put option where the holder receives a 10 year at the money put option upon shouting. The maximum contract maturity is 30 years. The contract has an initial strike $K_{0}=\$ 100$ and an initial maturity of 10 years. Black-Scholes modelling assumptions with $\sigma_{B S}=.25$ and $r=.06$.

at $S \approx \$ 100$ is about $\$ 35$ without the maturity extension feature but close to $\$ 40$ with it.

\subsection{Modelling The Deferred Payment Of Embedded Options}

As noted above, shout options are frequently embedded in other financial products, such as segregated funds. It is common practice in Canada to amortize the cost of the contract over its life by having the purchaser pay a percentage charge for the shout option feature.

The management operating expenses and insurance premiums of segregated funds are often paid indirectly by the fund itself. In this way, the value of an investment in a segregated fund is decreased by the the incurred operating expenses, usually on a daily basis. This can be modelled by considering the incurred expenses to be a dividend yield paid by the fund. Of course, the investor does not get to keep this dividend payment; instead it is paid to the fund manager and the insurance company.

In Figure 7 we see that as the expense rate, $r_{e}$, is increased the initial value of the maturity guarantee actually increases. The reason for this is simple; the maturity guarantee is required to cover any losses in the fund as well the premiums charged. Of course, the investor is not better off with a higher expense rate. If the guarantee ultimately proves worthless (because the underlying finishes above the guaranteed level), the value of the investor's account will be lower with a higher expense rate. Moreover, opportunities to reset the guarantee will come at lower levels of the underlying asset value given a higher expense rate.

Since these guarantees are often sold without an initial premium, a relevant task is to find the operating expense rate, $r_{e}$, which makes the present value of the payments to be 


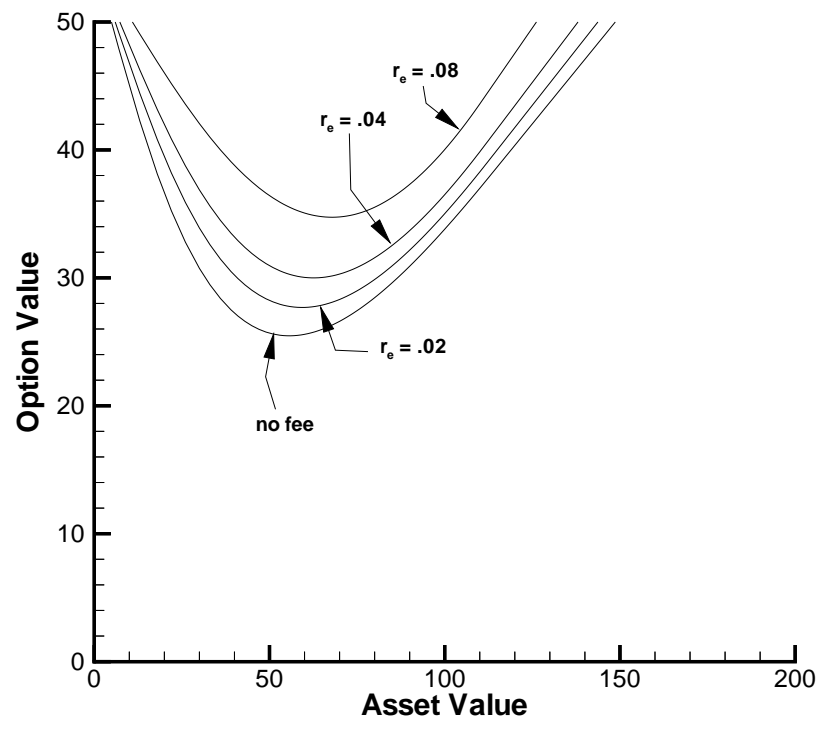

FIGURE 7: Value of a shout option with 2 shout opportunities per year (20 total), with an initial strike $K_{0}=\$ 100$, subject to various operating expenses, $r_{e}$. Notice that the initial value of the contract increases with the expense rate $r_{e}$ charged to the customer. Black-Scholes modelling assumptions with $\sigma_{B S}=.25, r=.06$, and $T=10$ years.

received equal to the initial contract value. This amortization of the payment for the shout option feature further complicates the hedging of these products. Receiving a proportional yield has the undesirable effect that less income is received when the value of the fund is low, which is exactly the time when the insurance policy is very valuable. As an extreme example, consider the case where value of the underlying asset goes to zero immediately after the sale of the maturity guarantee. Being charged a proportional amount, the investor actually receives this guarantee for nothing!

If the length of maturity of the contract $T$ is known, then under Black-Scholes assumptions it can be shown [11] that the proportional expense rate, $r_{e}$, which makes the investor indifferent to paying the guarantee premium, $V$, up front is given by

$$
V=S_{0}\left(1-e^{-r_{e} T}\right)
$$

In Table 3 we determine the net initial value of the contract to the writer of a ten year shout option (with two shout opportunities per year). We emphasize the difference between the value of the contract sold and the net value to the writer; the net value to the writer includes the benefit of receiving the stream of payments associated with the guarantee.

Finally, there exists the possibility that the holder will lapse on the contract leaving the writer with a hedging strategy which has only been partially paid for. Of course, this hedging portfolio has positive value (since it is replicating a non-negative set of outcomes). However, the value of this hedging portfolio may not be as much as the accumulated value of the received payments, resulting in a net loss for the writer. 


\begin{tabular}{|l|r|r|r|r|r|}
\cline { 2 - 6 } \multicolumn{1}{c|}{} & \multicolumn{5}{c|}{ Dollar Amount } \\
\cline { 2 - 6 } \multicolumn{1}{c|}{} & $r_{e}=.00$ & $r_{e}=.01$ & $r_{e}=.02$ & $r_{e}=.04$ & $r_{e}=.06$ \\
\hline Option value & 33.81 & 34.38 & 35.02 & 36.51 & 38.20 \\
\hline Equivalent up front payment & 0.00 & 9.52 & 18.13 & 32.97 & 45.12 \\
\hline Net Value & -33.81 & -24.86 & -16.89 & -3.54 & 6.92 \\
\hline
\end{tabular}

TABLE 3: The net initial value to the writer of writing a shout option at various operating expense rates, $r_{e}$, at asset level $S=\$ 100$. The shout option has two shout opportunities per year (20 total) and an initial strike $K_{0}=\$ 100$. Black-Scholes modelling assumptions with $\sigma_{B S}=.25, r=.06$, and $T=10$ years.

\subsection{The Effects On Contract Value Of Some Alternative Specifi- cations}

We can see by the examples presented so far that these option contracts may be quite valuable. Essentially, this is a result of the fact that these contracts can never be thought of as being deep out of the money. If the current guarantee level is unlikely to be used at expiry, the investor can reset to a better guaranteed level which is more likely to be worth money at expiry. We can think of these contracts as having value coming from two sources: (a) from the payoff of the presently held guarantee level; and (b) from the ability to shout an reset the guarantee to a higher level. In Figure 3(a) we can see these two regions by comparing the value of the shout option with a standard European put (which has no "shout" value) and a shout option with no initial strike setting (which has no "current guarantee" value).

There are several potential ways to modify these types of contracts so as to reduce their value. One possibility is to place restrictions on the availability of the shout opportunities. For instance, segregated funds are commonly sold with the ability to shout two or four times per year. In Figure 8, we can see that this does reduce the value of the option, though the decrease is perhaps not as dramatic as might be expected.

We proceed further by restricting the shout opportunities to fixed dates. Shown in Figure 8 is an option where the holder is only allowed to shout on six month anniversaries of the sale of the contract. This results in a somewhat larger decrease in the value of the contract. The figure also shows that an even bigger effect can be achieved through a reduced percentage guarantee.

Along somewhat different lines, another possibility is to assume a default risk for the writer. Figure 8 also plots the value of an option under the assumption that the writer will not honour the contract if the underlying asset value at expiry falls to $50 \%$ of the protective floor setting. Clearly, there is a large drop in the contract value, since we are effectively capping the payoff (thus eliminating the states where the guarantee is most valuable). We make no claims here about whether or not this is realistic. Our point is simply that estimated values for these contracts may considerably overstate their true worth to investors, if there is a chance of default. 


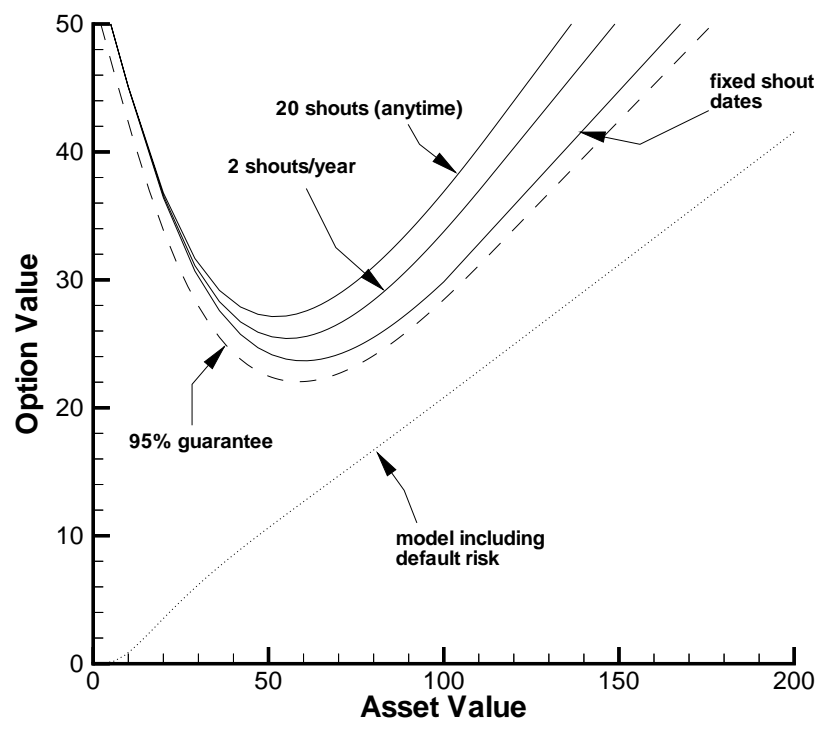

FiguRE 8: The impact of various contract features on the value of a shout option. Shown are an option with 20 shout opportunities available at any time during the life of the contract, an option which allows two shouts per year (20 total), and an option where the 20 shout opportunities are restricted to specific dates (half-yearly interval). All of these options have an initial strike of $K_{0}=\$ 100$ and reset the strike according to $K^{*}=S$. Slightly cheaper is an option with 2 shouts per year which provides an initial strike of $K_{0}=\$ 95$ and provides $95 \%$ guarantee protections, $K^{*}=.95 \mathrm{~S}$. Also shown is a model for a shout option which allows 2 shouts per year (anytime) and incorporates the default risk of the writer by assuming that the contract will not be honoured if the asset level falls from the guarantee level by 50\% upon expiry. Black-Scholes modelling assumptions with $\sigma_{B S}=.25$, $r=.06$, and $T=10$ years.

\subsection{Hedging Strategies And Considerations}

Strategies for hedging the risk exposure which arises from writing an option depend on the delta $(\Delta=\partial V / \partial S)$ and gamma $\left(\Gamma=\partial^{2} V / \partial S^{2}\right)$ of the particular contract. We have already seen that a shout option can be thought of as the opportunity to receive one of a collection of simpler options. As a result, once the numerical aspects of interpolation and discretization errors have been reduced to an acceptable level, the dynamic hedging strategy for the shout option will be as accurate as the valuation/hedging model for the building block European options.

Figure 9(a) shows the option delta for a standard European put option along with a single shout contract and a five shout contract. The general pattern is quite similar for all of these contracts. As the underlying asset value increases, the option values all become linear (but with a positive slope for the shout options, and steeper for five shout case than the single shout case). Given this observation, the option gammas will obviously all go to zero if the asset value gets large enough. Figure 9(b) shows that there isn't a great deal of difference 


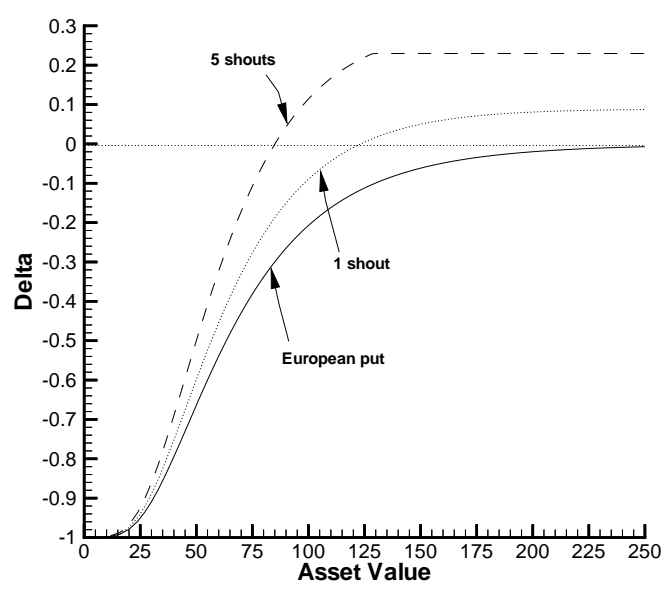

(a) Delta $(\Delta=\partial V / \partial S)$.

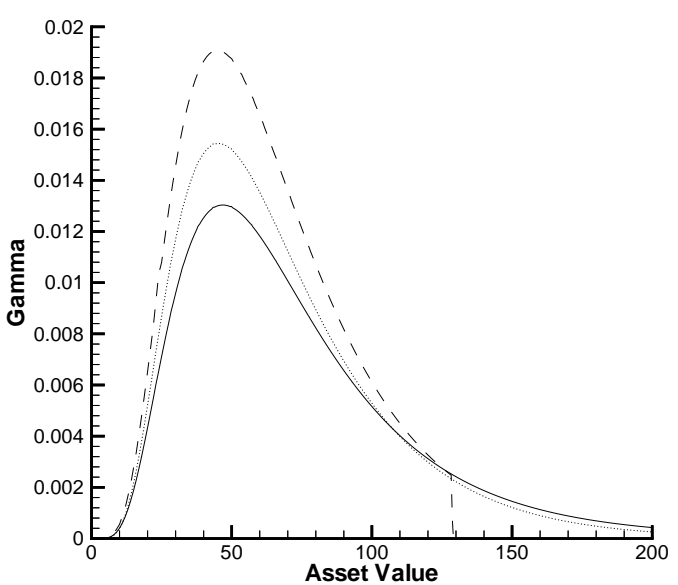

(b) Gamma $\left(\Gamma=\partial^{2} V / \partial S^{2}\right)$.

FiguRE 9: Shout option hedging parameters. Black-Scholes modelling assumptions with $\sigma_{B S}=.25, r=.06$, and $T=5$ years.

between the gammas of a standard European put and a single shout option contract. The five shout contract exhibits broadly similar behaviour, except near asset levels of approximately $\$ 130$, where its gamma falls very rapidly to zero. The general implication from these pictures is that the shout options are probably not significantly harder to dynamically hedge than a standard European put. ${ }^{6}$ Of course the long term nature of the contracts involved raises issues regarding the suitability of such an approach in the first place, since the assumption of constant parameters $(r, \sigma)$ is highly questionable in this context [3].

\subsection{Delta Hedged Value Versus Real Option Value}

In the context of a discussion involving long term protective floor contracts in the U.S., a portfolio manager was recently quoted as saying: "There's never been a 10-year window in which we would have had to pay out in the history of the S\&P 500". ${ }^{7}$ This suggests that writing long term puts on the market is fairly safe since it is unlikely that the contracts will end up in the money. Whatever its merits, this argument ignores the reset feature of shout option contracts. As noted above, such contracts are really never far from being in the money. Moreover, some concerns have been expressed regarding whether the institutions writing such options in Canada have appropriately hedged the risks involved. ${ }^{8}$

Suppose we assume that many of the shout options embedded in segregated funds have not been delta hedged. In this case, we can regard the embedded option as a type of "real

\footnotetext{
${ }^{6}$ Recall, however, that Figure 5 indicated that these contracts may become harder to hedge as the time until expiry decreases.

${ }^{7}$ Steve Killian, senior portfolio manager and chief operating officer, Mainstay Equity Index Fund, quoted in "New Aetna fund offers a guarantee, at a price", The Wall Street Journal, August 25, 1999. Mr. Killian did go on to observe that there is no reason to assume that history will necessarily repeat itself.

${ }^{8}$ See W. Falloon, “Canada's option nightmare”, RISK, August 1999.
} 
option". We can calculate the expected value of the contracts (rather than their delta hedged value) by solving the backward equation (see, e.g. [13]),

$$
\frac{\partial V}{\partial t}+\bar{\mu}(t) S \frac{\partial V}{\partial S}+\frac{1}{2} \sigma(S, t)^{2} S^{2} \frac{\partial^{2} V}{\partial S^{2}}-r(t) V=0
$$

where the risk adjusted drift rate, $\bar{\mu}$, is not replaced by its risk neutral counterpart $r$. The risk adjusted drift rate $\bar{\mu}$ reflects risk preferences [13]. We will refer to the value of this option as the "present expected value" of the contract. An inspection of equation (10) shows that the present expected value of a standard European put option is less than its delta hedged (Black-Scholes) value when $\bar{\mu}>r$. Intuitively, this is because if $\bar{\mu}>r$, the asset price is more likely to drift out of the money by expiry.

The interesting result is that the value of a shout option does not show a decrease in value to anywhere near the same extent. The value of a shout option comes from two sources, the payoff of the initial guarantee and the ability to lock in at higher levels during the life of the contract. At the money, $S=\$ 100$, the majority of the value comes from the ability to shout and lock in at higher asset levels, not from the initial guarantee. With the higher drift rate one expects to be able to lock in at a higher level, offsetting the decrease in value of the put option. In Figure 10 we see that the decrease in value of the shout put option is minimal compared with the decrease in value of a standard European put option when $S \approx \$ 100$.

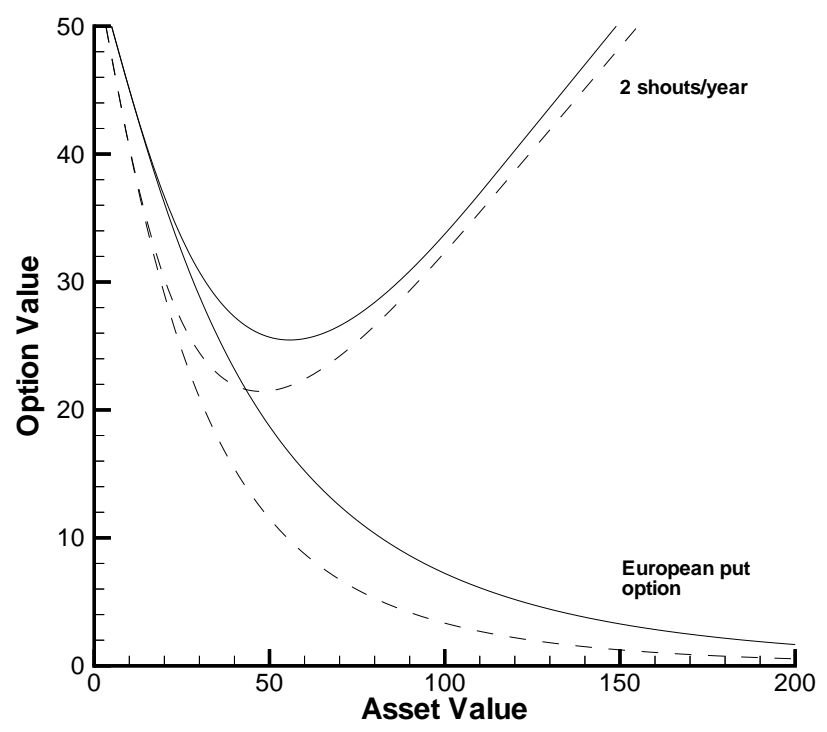

FiguRE 10: Comparison of present expected value (dashed) with the delta hedged BlackScholes value (solid). The shout option contract allows two shouts per year (total=20). Notice that at the money, $S=\$ 100$, the present expected value of the European put option shows a dramatic decrease in value compared with the delta hedged value. By contrast, the present expected value of the shout option is relatively close to its delta hedged value. Parameters used: $\sigma_{B S}=.25, r=.06, \bar{\mu}=.10, K_{0}=\$ 100, T=10$ years. 


\subsection{Uncertain Parameters}

As noted above, the assumption of constant parameters (such as $r$ and $\sigma$ ) is highly questionable in the context of long term option valuation. Ideally, it would be desirable to explore stochastic volatility or stochastic interest rate models, but this is not feasible given the complexity of the contracts (recall that we are already solving a two or three dimensional problem, and that including the feature of resetting the time until expiry in addition to the strike adds another dimension).

An alternative is to consider the use of uncertain parameter models $[2,15]$. In this context, we specify a range over which a parameter is assumed to vary throughout the life of the contract. The downside of this approach is that we do not end up with a single option value, but rather a range of possible values (from "best-case" to "worst-case"). At first glance, this might seem like a trivial idea which simply involves calculating the option values for the postulated high and low values of the parameter. However, this implicitly assumes that the option value function is monotonic with respect to the parameter. If this is not the case, then we are actually faced with solving a more general (and non-linear) PDE. Note that these models do not admit the similarity reduction described previously and we are forced to solve the full three dimensional problem. See [15] for further details regarding uncertain parameter models.

With regard to a shout put option, we observe in Figure 9(b) that the gamma is always positive. This implies that in an uncertain volatility model the best and worst cases will be given by the high and low values of the assumed range for $\sigma$. However, we can see in Figure 9 (a) that the delta of the shout option is not strictly negative as it is for a vanilla European put. This may require that the hedging strategy switches between long and short positions in the underlying asset at different times, making it more difficult to obtain best and worst case bounds for the value of the contract if interest rates or dividend yields (since these parameters multiply the option delta in the PDE) are uncertain.

Suppose we are willing to assume that the dividend yield lies within the range

$$
q^{-} \leq q \leq q^{+}
$$

We can obtain a bound for the best-worst cases by solving a non-linear generalization of the PDE. This uncertain dividend yield model uses

$$
\begin{gathered}
q_{\text {best }}(\Delta)=\left\{\begin{array}{ll}
q^{+} & \text {if } \Delta<0 \\
q^{-} & \text {if } \Delta \geq 0
\end{array},\right. \\
q_{\text {worst }}(\Delta)=\left\{\begin{array}{ll}
q^{+} & \text {if } \Delta>0 \\
q^{-} & \text {if } \Delta \leq 0
\end{array},\right.
\end{gathered}
$$

for the best and worst cases of the long position respectively. We reiterate that the best and worst cases for uncertain dividends cannot be determined by using the solutions for the extreme values of the dividend rates since the option delta is not of constant sign.

Figure 11(a) shows the results for an illustrative case where the dividend yield $q$ is assumed to lie within the range 1\%-5\%. The solid lines depict the best/worst case envelope, whereas the dotted lines show the option values obtained when we assume that the dividend 


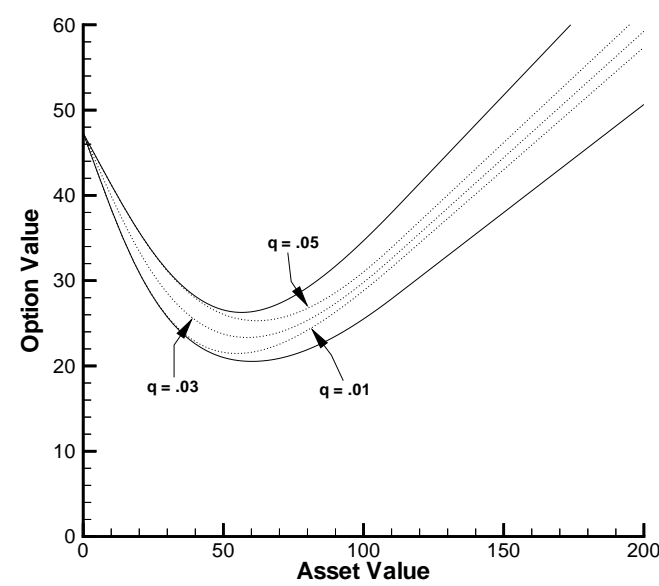

(a) Uncertain dividend yield case: $r=$ $.075, .01 \leq q \leq .05$.

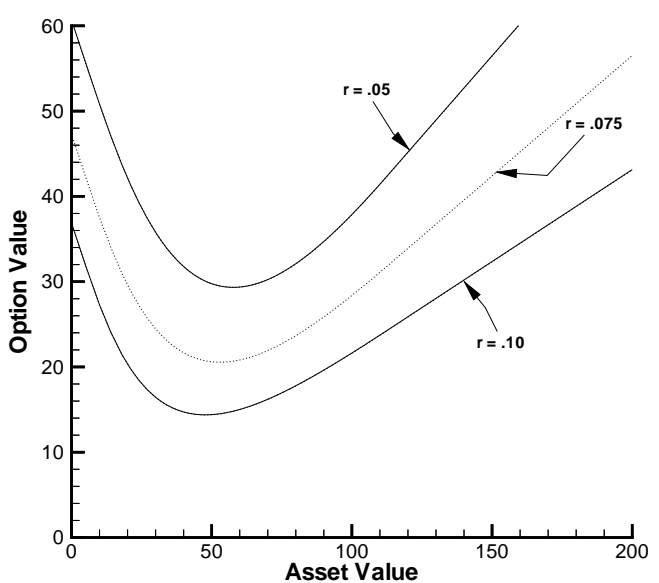

(b) Uncertain interest rate case: $q=0$, $.05 \leq r \leq .10$

FiguRE 11: Uncertain parameter models. The solid lines represent the best/worst case envelope. The contract allows two shouts per year (total $=20)$; Parameters used: $\sigma_{B S}=$ $.25, K_{0}=\$ 100$, and $T=10$ years.

yield is $1 \%, 3 \%$, and $5 \%$. Note that the option value increases with $q$ for these constant parameter cases. However, due to the non-linear nature of the uncertain parameter specification, the best/worst case envelope lies outside the option values calculated for $q=1 \%$ and $q=5 \%$. Moreover, the range in values is quite wide, indicating that the computed values are quite sensitive to the assumption that the dividend yield is known with certainty.

We now turn to examining the case of uncertain interest rates. The terms involving $r$ in the PDE are $r(S \Delta-V)$. It can be shown that $S \Delta-V$ is always non-positive for these contracts. ${ }^{9}$ As a result the envelope generated by the best and worst cases is given by the extreme values of the interest rate range. Due to the long term nature of these option contracts, the effect of interest rates on their value is extremely important. This is illustrated in Figure 11(b), which shows that near the money $(S \approx \$ 100)$, the option value with an interest rate of $5 \%$ is close to double that with an interest rate of $10 \%$.

We conclude this section by observing that alternative specifications such as constant elasticity of variance (CEV) models [8] can be easily handled in our general numerical framework. This is potentially of interest since recent research [5, 9] has reported that computed prices of some types of exotic options (e.g. barriers, lookbacks) for CEV models are much more sensitive to departures from the Black-Scholes setting than are prices of standard option contracts. Of course, this also means that hedging strategies are substantially different across the CEV class of models for these exotic options. Results indicating that this is also true in the context of shout options are provided in [16].

\footnotetext{
${ }^{9}$ To see this, consider the case where no initial strike is set. The option value in this situation is linear in $S$ and passes through the origin. Therefore, for such a contract $S \Delta-V$ is always zero. Also recall that as $S$ gets large, contracts where an initial strike is set become equivalent to the case of no initial strike (Figure 3(b)). Since the delta of these contracts is non-decreasing in $S$ (Figure $9($ a)), we have $S \Delta-V \leq 0$.
} 
If even further generality is desired, it is also easy to incorporate an implied volatility surface $[1,7]$ into our algorithm. In the case of a simple shout option the contract received upon shouting is a vanilla option. The volatility surface can be viewed as a method of interpolating the prices of traded options to increase the consistency of the model with presently observed market prices.

\section{Conclusions}

Shout options are a general class of financial contracts which allow the holder to modify the contract. We can model these products by solving a system of PDEs which satisfy minimum value constraints. We present an object-oriented framework which provides the necessary flexibility to model complex contract designs as well as incorporate various economic modelling techniques. We extend this framework to a parallel version capable of exploiting modern high performance multiprocessor computer architectures. The performance gains achieved compare favourably with theoretical expectations. It is worth emphasizing again that many other exotic options can be valued using similar techniques. An interesting avenue for future research involves the application of high-performance computing to these types of financial valuation problems.

In this paper we have explored the valuation of some complicated types of shout options under various modelling assumptions. Given the size of the Canadian segregated fund market $\left(\$ 60\right.$ billion $\left.^{10}\right)$, this is a problem of considerable practical interest for option writers and regulators alike. We have described some of the difficulties in hedging these contracts, and illustrated the effects of different contract provisions on their values. We have also seen how the valuation of these options is very sensitive to various modelling assumptions. If one simply uses Black-Scholes assumptions with constant parameters, it is quite possible (even likely) that the contracts will be considerably mispriced and incorrectly hedged.

\section{References}

[1] L.B.G. Andersen and R. Brotherton-Ratcliffe. The equity option volatility smile: An implicit finite difference approach. Journal of Computational Finance, 1(2):5-37, 1998.

[2] M. Avellaneda, A. Levy, and A. Parás. Pricing and hedging derivative securities in markets with uncertain volatilities. Applied Mathematical Finance, 2:73-88, 1995.

[3] G. Bakshi, C. Cao, and Z. Chen. Pricing and hedging long-term options. Journal of Econometrics, forthcoming, 1999.

[4] P.P. Boyle and M.R. Hardy. Reserving for maturity guarantees: Two approaches. Insurance: Mathematics \& Economics, 21:113-127, 1997.

[5] P.P. Boyle and Y. Tian. Pricing lookback and barrier options under the CEV process. Journal of Financial and Quantitative Analysis, 34:241-264, 1999.

\footnotetext{
${ }^{10}$ W. Falloon, "Canada's option nightmare", RISK, August 1999.
} 
[6] T.H.F. Cheuk and T.C.F. Vorst. Shout floors. Net Exposure, 2, November 1997.

[7] T.F. Coleman, Y. Li, and A. Verma. Reconstructing the unknown local volatility function. Journal of Computational Finance, 2(3):77-102, 1999.

[8] J.C. Cox. Notes on option pricing I: Constant elasticity of variance diffusions. Working paper, Stanford University (reprinted in Journal of Portfolio Management 22 (1996), 15-17), 1975.

[9] D. Davydov and V. Linetsky. Pricing options on one-dimensional diffusions: A unified approach. Working paper, University of Michigan, 1999.

[10] C. Johnson. Numerical Solutions of Partial Differential Equations by the Finite Element Method. Cambridge University Press, Cambridge, 1987.

[11] M.A. Milevsky and S.E. Posner. Option-adjusted equilibrium valuation of guaranteed minimum death benefits in variable annuities. Working paper, Schulich School of Business, York University, 1999.

[12] B. Thomas. Something to shout about. RISK, 6:56-58, May 1993.

[13] L. Trigeorgis. Real Options. MIT Press, Cambridge, 1996.

[14] K.R. Vetzal and P.A. Forsyth. Discrete Parisian and delayed barrier options: A general numerical approach. Advances in Futures and Options Research, 10:1-15, 1999.

[15] P. Wilmott. Derivatives. John Wiley and Sons, West Sussex, England, 1998.

[16] H. Windcliff, P.A. Forsyth, and K.R. Vetzal. Shout options: A framework for pricing contracts which can be modified by the investor. Working paper, University of Waterloo, http://ww.scicom. uwaterloo.ca/ paforsyt/shoutnum.ps, 1999.

[17] R. Zvan, P.A. Forsyth, and K.R. Vetzal. Penalty methods for American options with stochastic volatility. Journal of Computational and Applied Mathematics, 91:119-218, 1998.

[18] R. Zvan, P.A. Forsyth, and K.R. Vetzal. Discrete Asian barrier options. Journal of Computational Finance, 3(1):41-67, 1999. 